\title{
Severe agitation following deep brain stimulation for parkinsonism
}

\author{
Nicholas G.W. Rose, MD, PhD ${ }^{*}$; Michael Mostrenko, $\mathrm{MD}^{\dagger}$; Jacqueline McMaster, BMed ${ }^{\ddagger}$; \\ Christopher R. Honey, MD, DPhil ${ }^{\ddagger}$
}

\section{ABSTRACT}

The use of deep brain stimulation has become increasingly common for the treatment of movement disorders, including Parkinson disease. Although deep brain stimulation is generally very successful in alleviating the extrapyramidal symptoms of Parkinson disease, side effects can occur. This case report describes a patient presenting to the emergency department in a state of extreme aggression 3 days after a change in the parameters of his bilateral subthalamic nucleus stimulator. We review the complications of deep brain stimulation relevant to the emergency physician and provide some practical information on stimulator adjustment in an emergency.

\section{RÉSUMÉ}

Il est de plus en plus courant d'utiliser la stimulation cérébrale profonde pour traiter les troubles du mouvement, y compris la maladie de Parkinson. Bien que cette technique donne généralement de très bons résultats pour atténuer les symptômes extrapyramidaux de la maladie de Parkinson, des effets secondaires peuvent se produire. Nous présentons le cas d'un patient qui s'est présenté à l'urgence dans un état d'extrême agressivité trois jours après un changement aux paramètres des électrodes de stimulation bilatérale du noyau sous-thalamique. Nous passons en revue les complications de la stimulation cérébrale profonde pertinentes pour le médecin d'urgence et fournissons de l'information pratique sur le réglage du stimulateur en urgence.

Keywords: complications, deep brain stimulation, Parkinson disease, subthalamic nucleus

Deep brain stimulation (DBS) is becoming increasingly common for the treatment of extrapyramidal movement disorders, especially in patients with Parkinson disease. ${ }^{1,2}$ The technique is also used for chronic pain syndromes and in research protocols involving depression, ${ }^{3}$ Tourette syndrome, ${ }^{4}$ and epilepsy. ${ }^{5}$ For the treatment of Parkinson disease, the procedure involves electrodes inserted into the brain and connected via subcutaneous wires to an implantable neural stimulator (INS), like a pacemaker, in the chest (Figure 1). The most common stimulation target in Parkinson disease is the subthalamic nucleus (STN). The INS can be switched on and off, and adjusted, via a remote control termed the access review therapy controller (ARTC). As of January 1, 2009, three main types of INS were in use: the single electrode Itrel 3 and Soletra and the magnetically shielded double-electrode Kinetra (all from Medtronic, Minneapolis, MN), used in bilateral STN-DBS. DBS has been shown to effectively suppress tremor and rigidity as well as akinesia and dyskinesia in Parkinson disease. ${ }^{1}$

A variety of complications have been described and can present at any time after the initial electrode placement. ${ }^{6}$ DBS patients can present to the emergency department (ED) with infection, battery exhaustion, hardware failure, and the side effects of stimulation, which include stimulation-induced dyskinesias and behavioural problems.

\section{CASE REPORT}

A 76-year-old man with an 8-year history of progressive Parkinson disease despite maximum medical therapy was brought to the ED after sudden onset

Department of Emergency Medicine, Vancouver General Hospital, University of British Columbia, Vancouver, BC; †Department of Emergency Medicine, Royal Columbian Hospital, New Westminster, BC; $¥$ Division of Neurosurgery, Department of Surgery, Faculty of Medicine, University of British Columbia, Vancouver, BC.

Correspondence to: Dr. Nicholas G.W. Rose, Department of Emergency Medicine, Vancouver General Hospital, 855 West 12th Avenue, Vancouver, BC; nrose@interchange.ubc.ca.

This article has been peer reviewed.

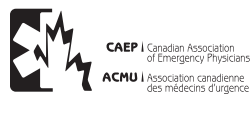




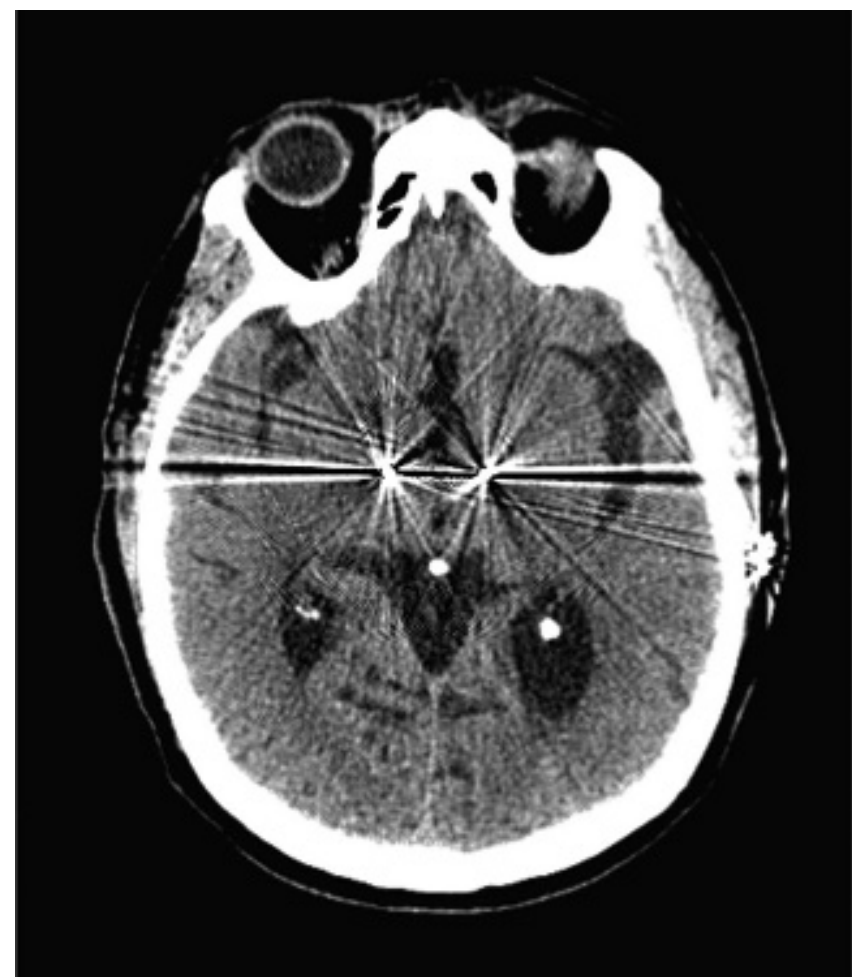

Figure 1. Deep brain stimulator electrodes in the subthalamus.

of increasingly aggressive behaviour over a 6-hour period. Three months previously, the patient had a DBS placed with regular neurosurgical follow-up, including a visit to the DBS clinic 3 days prior to his presentation to the ED. During this visit, neurostimulation had been increased to try to compensate for the progression of the patient's "freezing" episodes. The adjusted settings were an amplitude of $2.0 \mathrm{~V}$ bilaterally, a pulse width of 90 microseconds, and a frequency of $135 \mathrm{~Hz}$ on both right and left sides with monopolar stimulation using the most superficial contact on the left and second most superficial contact on the right.

The patient's other medical history included a diagnosis of bowel cancer 7 years previously with a recent recurrence and Hodgkin's lymphoma diagnosed 2 years previously and currently in remission. He had also suffered a non-ST segment elevation myocardial infarction 2 years previously and had known hyperlipidemia. His medications were levodopa-carbidopa IR 100/25 mg once daily; levodopa-carbidopa CR $100 / 25 \mathrm{mg}$, one tablet at $4 \mathrm{am}$ and noon, half-tablet at 9 am and $10 \mathrm{pm}$, quarter-tablet at $4 \mathrm{pm}$; bromocriptine $5 \mathrm{mg}$ four times daily; metoprolol $25 \mathrm{mg}$ twice daily; doxazosin $4 \mathrm{mg}$ at bedtime; simvastatin $20 \mathrm{mg}$ daily; and aspirin $81 \mathrm{mg}$ daily. There had been no recent changes in his medication. The patient had no history of alcohol or recreational substance abuse and no previous psychiatric disorder.

The patient was sent to the ED from his nursing home after suddenly becoming aggressive toward other residents, entering their rooms and uttering profanities at them for no apparent reason. This behaviour was entirely out of keeping with the patient's normal demeanour. $\mathrm{He}$ was initially easily redirected but then began hiding in closets and startling nursing home residents and staff. Increasing movement and gait difficulties were also noted. When he became increasingly agitated, hurled himself to the floor, and chewed through the call bell cord, an ambulance was called. The police were also called to assist because he became physically violent, and the patient was transferred to hospital.

The patient presented to the ED at $2330 \mathrm{~h}$. He was alert but noncooperative with a detailed mental status examination. His temperature was $36.0^{\circ} \mathrm{C}\left(96.8^{\circ} \mathrm{F}\right)$ orally and blood pressure was $132 / 88 \mathrm{~mm} \mathrm{Hg}$ with a heart rate of 72 beats per minute. His respiratory rate was 16 breaths per minute, and his oxygenation was $96 \%$ on room air. Blood sugar performed by paramedics was $4.4 \mathrm{mmol} / \mathrm{L}$. The patient appeared agitated but was generally cooperative and denied any focal injury or pain. He had dyskinetic movements and was writhing in the stretcher. No localizing abnormalities were identified on cranial nerve and focused neurologic examination. The remainder of the physical examination was unremarkable. Complete blood count and serum electrolyte, creatinine, and liver tests were within the normal range.

The neurosurgical team following the patient for the DBS was contacted, and the suggestion was made to turn the electrode stimulator to the lowest setting $(1.0 \mathrm{~V}$ bilaterally). By using the access controller, the INS unit was easily adjusted at the bedside by the emergency physician. The dyskinetic movements stopped immediately, and the patient slumped into bed, falling asleep shortly thereafter. He was easily arousable from sleep. Owing to the remarkable reversal after the DBS unit was turned to its lowest setting and the patient's subsequent normal neurologic examination, no further investigations were performed, and the patient was subsequently discharged back to the nursing home with follow-up in the neurostimulator clinic. The patient subsequently suffered further episodes of DBS-induced agitation that eventually resolved with further neurostimulator and medication adjustments. 


\begin{tabular}{l} 
Table 1. Postoperative complications of implantable neural \\
stimulator insertion \\
\hline Postsurgical complications \\
Hemorrhage (immediate) \\
Seizures (immediate) \\
Infection (0-6 mo) \\
Erosions (0-12 mo) \\
Stimulation side effects \\
Psychiatric \\
Depression/suicidality \\
Hypomania/euphoria \\
$\quad$ Anxiety \\
Hyperstimulation/aggression \\
Neurologic \\
Diplopia \\
Ataxia \\
$\quad$ Paresthesias \\
Rigidity/tremors \\
Hardware \\
Battery failure \\
Lead fracture/migration \\
Inadvertent on/off \\
Non-DBS-related emergencies \\
MRI exposure \\
ECG interpretation \\
Electrocautery/diathermy \\
Defibrillation \\
\hline DBS = deep brain stimulation; ECG = electrocardiogram; MRI = magnetic resonance \\
imaging. \\
\hline
\end{tabular}

\section{DISCUSSION}

DBS can improve the symptoms of advanced Parkinson disease considerably. However, like any surgical procedure with hardware placement, a number of complications can occur. The postoperative complications are listed in Table 1.

\section{Postoperative complications}

Most postsurgical complications occur during the immediate postoperative period while in hospital. A recent review describes a complication rate of 3 to $6 \%$ for intracranial hemorrhage and 4 to $10 \%$ for seizure. ${ }^{3}$ None of these events occurred out of hospital. Infection (chest $70 \%$, scalp 30\%, brain $0 \%$ ) has been reported to occur in 2 to $15 \%$ of implantations up to 5 months postoperatively; however, the vast majority occur within 1 month of surgery..$^{4,7,8}$ Skin flora, most often Staphylococcus aureus, are the most common organisms isolated. Prompt antibiotic coverage and neurosurgical referral are essential. Skin erosions occurring anywhere along the path of the DBS device are often associated with infection and generally occur from 1 to 12 months postimplantation. ${ }^{6}$

\section{Hardware failure}

These types of malfunctions include battery failure, lead fracture and migration, and inadvertent switching off the INS unit. Most hardware failures are not emergencies and result in a patient's progressive return to pre-DBS symptoms. However, a portion of patients with advanced Parkinson disease may become so unwell that urgent referral and management by the neurosurgical team are required. Typical battery life of the INS is between 2 and 5 years. Lead migration can be assessed through imaging and occurs in up to $5 \%$ of patients, usually between 6 and 36 months after surgery. ${ }^{10}$ Lead fracture has been reported to occur in $5 \%$ of patients and generally occurs proximal to the INS, between 6 and 24 months after placement. ${ }^{11}$ This is uncommon without a history of trauma.

A careful history is required to determine if the INS has been inadvertently shut off. Devices that cause strong electromagnetic fields, such as retail theftdetector gates, security gates at airports, high-voltage lines, and magnetic resonance imagers, as well as common household items that contain magnets (such as stereo speakers, cellular phones), have been known to inadvertently turn off the INS unit. ${ }^{12}$ This is less common with the new magnetically shielded INS. Most patients possess an ARTC, which can be used to determine the on/off status of the INS (Appendix).

\section{Stimulation side effects}

This case report describes a stimulation-induced side effect of DBS. The resulting signs and symptoms of inappropriate stimulation may be recognized when they differ from the condition being treated by DBS. A variety of negative effects have been described, including depression, ${ }^{13}$ suicidality, ${ }^{14}$ apathy, ${ }^{15}$ mania, ${ }^{16}$ anxiety, ${ }^{17}$ hypersexuality, ${ }^{18}$ and aggressiveness. ${ }^{19}$ These symptoms generally occur soon after adjustment of the DBS and thus may occur months to years after implantation. If the symptoms are sufficient to require intervention, consultation with the neurostimulator team should be sought. The INS can be reduced to its lowest setting using the ARTC or shut off using either the ARTC or a magnet if the magnet control switch 
has not been disabled. It may take hours for the adverse DBS-induced symptoms to resolve.

\section{Non-DBS emergencies}

Non-DBS emergencies are usually related to nonneurologic conditions where the presence of the INS may interfere with the presenting complaint. Electrocardiogram (ECG) interpretation may be impossible owing to artifact from a unipolar INS..$^{20}$ Temporarily turning the unit off or switching to a bipolar mode can eliminate the problem. ${ }^{21}$

Cardioversion for atrial fibrillation at 100 and 200 $\mathrm{J}$ in a patient with an older model thalamic INS (no longer in production), with the paddles placed over the right chest and apex of the heart, resulted in lesioning of the thalamus with peripheral paresthesias, allodynia, and dysesthetic pain syndrome..$^{22}$ Another report describes a patient with an implantable cardioverter-defibrillator who suffered repeated defibrillations but remained neurologically asymptomatic. ${ }^{9}$ It is recommended that the INS voltage be turned off before cardioversion, ${ }^{9}$ but this should not delay emergency defibrillation. Additionally, the pads should be placed as far away as possible from the INS, ideally in the anterior/posterior position because most INSs are placed in the left chest wall.

Magnetic resonance imaging (MRI) has been safely performed in patients who have a DBS. ${ }^{9}$ However, the manufacturer recommends imaging under specific conditions owing to concerns about producing heating at the electrode-tissue interface in the brain..$^{23}$ The treating DBS team should be contacted prior to any MRI.

The manufacturer recommends avoidance of electrocautery in these patients. Monopolar electrocautery can cause electric shocks without any other neurologic sequelae. ${ }^{24}$ It is recommended that the INS be turned off before electrocautery and the dispersive plate placed so that the electrical field does not cross the DBS system. Bipolar electrocautery has reportedly not caused any adverse effects in patients. ${ }^{12,24}$

Diathermy (deep heat therapy) is absolutely contraindicated in DBS patients because heat may be conducted through the system. Two patients who were exposed to diathermy in the maxilla after tooth extraction $^{25}$ and for treatment of chronic scoliosis ${ }^{26}$ developed a permanent vegetative state.

\section{CONCLUSION}

The use of deep brain and spinal cord stimulation devices is rising as they are used to treat Parkinson disease, chronic pain, and chronic depression. The emergency physician should be aware of the DBS, its side effects, and the measures needed to inactivate it.

Competing interests: None declared.

\section{REFERENCES}

1. Deuschel G, Schade-Brittinger C, Krack P, et al. A randomized trial of deep-brain stimulation for Parkinson's disease. N Engl 7 Med 2006;355:896-908.

2. Hallett MM, Litvan I. Evaluation of surgery for Parkinson's disease: a report of the Therapeutics and Technology Assessment Subcommittee of the American Academy of Neurology. The Task Force on Surgery for Parkinson's Disease. Neurology 1999;53:1910-21.

3. Mayberg H, Lozano A, Voon V, et al. Deep brain stimulation for treatment-resistant depression. Neuron 2005;45:651-60.

4. Servello D, Porta M, Sassi M, et al. Deep brain stimulation in 18 patients with severe Gilles de la Tourette syndrome refractory to treatment: the surgery and stimulation. 7 Neurol Neurosurg Psychiatry 2008;79:136-42.

5. Theodore $W$, Fisher R. Brain stimulation for epilepsy. Lancet Neurol 2004;3:111-8.

6. Seijo FJ, Alvarez-Vega MA, Gutierrez JC, et al. Complications in subthalamic nucleus stimulation surgery for treatment of Parkinson's disease. Review of 272 procedures. Acta Neurochir (Wein) 2007;149:867-76.

7. Sillay KA, Larson PS, Starr PA, et al. Deep brain stimulator hardware-related infections: incidence and management in a large series. Neurosurgery 2008;62:360-7.

8. Temel Y, Ackermans L, Celik H, et al. Management of hardware infections following deep brain stimulation. Acta Neurochir (Wein) 2004;146:355-61.

9. Peña E, Pastor J, Hernando V, et al. Skin erosion over implants in deep brain stimulation patients. Stereotact Funct Neurosurg 2008;86:120-6.

10. Goodman RR, Kim B, McClelland S III, et al. Operative techniques and morbidity with subthalamic nucleus deep brain stimulation in 100 consecutive patients with advanced Parkinson's disease. 7 Neurol Neurosurg Psychiatry 2006;77: $12-7$.

11. Hamani C, Lozano AM. Hardware-related complications of deep brain stimulation: a review of the published literature. Stereotact Funct Neurosurg 2006;84:248-51.

12. Blomstedt P, Jabre M, Bejjani B-P. Electromagnetic environmental influences on implanted deep brain stimulators. Neuromodulation 2006;9:262-9.

13. Bejjani BP, Damier P, Arnulf I, et al. Transient acute depression induced by high-frequency deep-brain stimulation. N Engl 7 Med 1999;340:1476-80.

14. Berney A, Vingerhoets F, Perrin A, et al. Effect on mood of subthalamic DBS for Parkinson's disease: a consecutive series of 24 patients. Neurology 2002;59:1427-9. 
15. Krack P, Batir A, Van Blercom N, et al. Five-year follow-up of bilateral stimulation of the subthalamic nucleus in advanced Parkinson's disease. N Engl 7 Med 2003;349: 1925-34.

16. Kulisevsky J, Berthier ML, Gironell A, et al. Mania following deep brain stimulation for Parkinson's disease. Neurology 2002;59:1421-4.

17. Houeto JL, Mesnage V, Mallet L, et al. Behavioural disorders, Parkinson's disease and subthalamic stimulation. 7 Neurol Neurosurg Psychiatry 2002;72:701-7.

18. Morgan JC, di Donato CJ, Jenkins PD, et al. Selfstimulatory behaviour associated with deep brain stimulation in Parkinson's disease. Mov Disord 2006;21:283-5.

19. Sensi M, Eleopra R, Cavallo MA, et al. Explosive aggressive behavior related to bilateral subthalamic stimulation. Parkinsonism Relat Disord 2004;10:247-51.

20. Martin WA, Camenzind E, Burkhard PR. ECG artifact due to deep brain stimulation. Lancet 2003;361:1431.
21. Constantoyannis C, Heilbron B, Honey CR. Electrocardiogram artifacts caused by deep brain stimulation. Can 7 Neurol Sci 2004;31:343-6.

22. Yamamoto T, Katayama Y, Fukaya C, et al. Thalamotomy caused by cardioversion in a patient treated with deep brain stimulation. Stereotact Funct Neurosurg 2000;74:73-82.

23. Medtronic. MRI guidelines for Medtronic deep brain stimulation systems. Available at: http://professional.medtronic.com/ wcm/groups/mdtcom_sg/@mdt/@neuro/documents/documents/ dbs-2007-mri.pdf (accessed Jan 2011).

24. Weaver J, Kim SJ, Lee MH, Torres A. Cutaneous electrosurgery in a patient with a deep brain stimulator. Dermatol Surg 1999;25:415-7.

25. Nutt JG, Anderson VC, Peacock JH, et al. DBS and diathermy interaction induces severe CNS damage. Neurology 2001;56:1384-6.

26. Medtronic. Important diathermy safety information. Minneapolis (MN): Medtronic; 2005. 